\title{
Effects of the Awareness-Driven Individual Resource Allocation on the Epidemic Dynamics
}

\author{
Xiaolong Chen $\mathbb{D D}^{1,2}$ Ruijie Wang, ${ }^{3}$ Dan Yang $\mathbb{D D}^{4,5}$ Jiajun Xian, ${ }^{4,5}$ and Qing $\mathrm{Li}^{1,2}$ \\ ${ }^{1}$ School of Economic Information Engineering, Southwestern University of Finance and Economics, Chengdu 611130, China \\ ${ }^{2}$ Financial Intelligence and Financial Engineering Key Laboratory of Sichuan Province, \\ School of Economic Information Engineering, Chengdu 611130, China \\ ${ }^{3}$ Aba Teachers University, Aba 623002, China \\ ${ }^{4}$ Department of Computer Science, School of Engineering, Shantou University, Shantou 515063, China \\ ${ }^{5}$ Key Laboratory of Intelligent Manufacturing Technology (Ministry of Education), Shantou University, Shantou 515063, China
}

Correspondence should be addressed to Dan Yang; danyangsjhd@hotmail.com

Received 18 August 2020; Revised 7 September 2020; Accepted 27 September 2020; Published 14 October 2020

Academic Editor: Chenquan Gan

Copyright (C) 2020 Xiaolong Chen et al. This is an open access article distributed under the Creative Commons Attribution License, which permits unrestricted use, distribution, and reproduction in any medium, provided the original work is properly cited.

\begin{abstract}
We investigate the effects of self-protection awareness on the spread of disease from the aspect of resource allocation behavior in populations. To this end, a resource-based epidemiological model and a self-awareness-based resource allocation model in complex networks are proposed, respectively. First of all, we study the coupled disease-awareness dynamics in complex networks with fixed degree heterogeneity. Through extensive Monte Carlo simulations, we find that overall the self-awareness inhibits the spread of disease. More importantly, the influence of the self-awareness on the spreading dynamics can be divided into three phases. In phase I, the self-awareness is relatively small and the outbreak of the epidemic can not be suppressed effectively. While, in phase II, the epidemic size is significantly reduced. Finally, in phase III, there is a sufficiently large value of self-awareness, the disease cannot outbreak anymore. Further, we study the impact of degree heterogeneity on the coupled disease-awareness dynamics and find that the network heterogeneity plays the role of "double-edged sword" in that it can either suppress or promote the epidemic spreading. Specifically, when the basic infection rate is relatively small, it promotes the spread of disease under the condition that there is a relatively small self-awareness. While, when the basic infection rate is relatively large, it inhibits the outbreak of epidemic at a relatively small self-awareness; in turn, it promotes the outbreak of epidemic at a relatively large selfawareness.
\end{abstract}

\section{Introduction}

From the Spanish Flu in Europe in the 1920s [1] to the SARS (Severe Acute Respiratory Syndrome) in 2003 [2] and the H1N1 flu outbreak in 2009 in the United States [3], the onset of each pandemic in the history always brought a tragic disaster to human beings. As of September 5, 2020, the ongoing COVID-19 outbreak has infected nearly 270 million people worldwide, and regrettably, more than 870,000 have lost their lives [4]. As a result, the mitigation and control of the spread of epidemics has always been a challenging subject for humans. During a pandemic, especially in the early stage of the epidemic, the individuals' self-protection awareness plays a vital role in the prevention and control of it [5]. The individuals can be well informed by the information about the status of the epidemic through social networking platforms and news media [6], which invokes the awareness of selfprotection. Subsequently, the individuals will take protective measures, such as wearing masks, reducing public gathering, and washing hands, to avoid being infected.

In recent decades, a large body of literatures have highlighted the critical role that human responses playing in influencing the spread of disease [7-10]. Driven by the awareness of self-protection, the individuals will take a series of precautionary measures to avoid infection $[11,12]$, and it will, in turn, affect the course of the epidemic by reducing the 
transmission of the disease. For example, due to the rapid response and the intensive control measures of both government and people in the early stages of the outbreak, the epidemic has been effectively controlled in China [13]. Consequently, the study of coupled dynamics of awareness and disease has been one of the most fruitful realms in various disciplines $[5,14,15]$. Typical representatives include the game theory in studying the vaccination behavior that the individuals decide whether to vaccinate or not based on an assessment of risks and benefits [16-18]. In addition to the application of game theory, the interplay between the awareness and epidemics in complex networks has attracted much attention in theoretical and empirical research [19-22]. The seminal work was the study of the spread of awareness in well-mixed populations and lattices and, in turn, its influence on the spread of disease by Funk et al. [7]. In addition to the well-mixed assumption, $\mathrm{Wu}$ et al. [23] explored, respectively, the impact of local, global, and contact awareness on epidemic spreading in networks with heterogeneous connections in populations. Moreover, based on the framework of multiplex networks, Granell et al. [24] investigated the coupled dynamics of the epidemic and awareness. Using a microscopic Markov chain approach and Monte Carlo simulations, they showed that the onset of the epidemics can be changed by the coupled dynamical process. Recently, Zhan et al. [25] studied the coupled dynamics of two different diseases and the corresponding information based on empirical analysis and theoretical modeling.

In addition to wearing masks, staying at home, etc., the behavior of resource donation in the individuals plays a vital role in disease control as the severe shortage of resources induced by the outbreak. For example, during the COVID19 pandemic, both personal protective and medical equipment such as respirators, gloves, and face shields are in severe shortage all over the world [26]. Consequently, the research of optimal allocation of both public and individual resources in controlling the spread of the disease has been one of the hottest topics in the past years [27-30]. For example, Preciado et al. [31] investigated the optimal allocation of vaccination resources during an outbreak of the disease in complex networks and found the cost-optimal strategy of resource allocation. Nowzari et al. [27] developed an optimization framework to solve the problems of finding minimum resource cost required to eradicate the disease and the optimal strategy of resource allocation based on the analysis of the proposed epidemiological model. In terms of individual resources, Böttcher et al. [32] investigated the impact of the shortage of individual resources induced by the outbreak of epidemics. They showed that the epidemics can spiral out of control if the recovery cost is higher than a critical value. Inspired by the work of Ref. [32], the coupled dynamics of resource allocation and disease spreading on both single and multiplex networks has been widely researched in recent years [33-37]. In spite of a large body of literatures about the interplay between awareness (resource) and epidemics, there is a lack of research on the co-evolutionary mechanism among the three dynamical processes.

To investigate the effects of the awareness-driven resource support of individuals on the epidemic dynamics, we propose a novel resource-based susceptible-exposed-infected-recovered (r-SEIR) epidemiological model and a selfawareness-based resource allocation model, respectively, in this paper. In the models, the self-protection awareness of a susceptible node (abbreviated to self-awareness) is supposed to be composed of both local and global awareness measured by the number of infected neighbors and global infected nodes, and the resource allocation probability of each node is determined by its awareness. First of all, we study the coupled disease-awareness dynamics by incorporating the individual resource allocation in complex networks with fixed degree heterogeneity. Through extensive Monte Carlo simulations, we find that the self-awareness of the individuals can inhibit the spread of disease. Specifically, we find two critical values of the instinctive self-awareness that separate the parameter space into three phases. In phase I, when there is a relatively small self-awareness, the outbreak size of the epidemic increases abruptly with the basic infection rate, which implies that the epidemic can not be suppressed effectively. While, in phase II, the final fraction of infected nodes increases slowly with the basic infection rate, which indicates that the disease can be controlled to a certain extent. Finally, in phase III, when there is a sufficiently large value of the self-awareness, the disease is well controlled. Next, we continue studying the effects of degree heterogeneity on the coupled dynamics. Through Monte Carlo simulations, we find that there is "double-edged sword" effect of network heterogeneity on the coupled dynamics. Specifically, when the basic infection rate is relatively small, the network heterogeneity promotes the spread of disease under the condition that there is a relatively small value of self-awareness. While, when the basic infection rate is relatively large, it inhibits the outbreak of epidemic at a relatively small self-awareness; in turn, it promotes the outbreak of epidemic at a relatively large self-awareness. Our findings can be applied directly to guide people to maintain the right level of self-protection awareness and take rational behaviors during a pandemic. Moreover, the results in this paper will also provide a constructive viewpoint for policymakers of public health.

\section{Model Description}

2.1. Epidemic Model. To investigate the impact of awarenessdriven individual resource allocation on the spread of the epidemic, we propose a resource-based susceptible-exposedinfected-recovered (r-SEIR) epidemiological model [38] in complex networks. The r-SEIR model is composed of the following four epidemiological compartments: susceptible $(S)$, exposed $(E)$, infected $(I)$, and recovered $(R)$. To facilitate the study of the dynamical processes, the individuals are represented by nodes in the network and an adjacency matrix $\mathbb{A}$ is introduced to store the information of network structure. If an edge between $i$ and $j$ exists, the matrix element $a_{i j}=1$; otherwise, $a_{i j}=0$. At each time step, the pathogen transmits from an I-state node to an S-state node at a basic infection rate $\beta$, if there is a contact between these two nodes and the $S$-state node does not take any protective measure. Those S-state nodes which get the pathogen will 
turn to E-state immediately. In the real scenario, any individual who gets the information about the disease will have awareness for self-protection. We consider that, initially, all nodes in the network have the same level of instinctive selfawareness, which is denoted as $\alpha$ in the context. With the development of the epidemic, they can get the information about the status of the epidemic from local communities or global mass media [23], which will alter the awareness of each individual. Based on the description above, it is assumed that the self-awareness of a node $i$, which is denoted as $\alpha_{i}$, is determined by both the number of I-state neighbors, denoted as $m_{i}(t)$, and the global confirmed cases, denoted as $I(t)$, in the complex network. Since the neighbor's infection of a node has a more intuitive effect on its self-awareness, a coefficient $\eta \in[0,1]$ is introduced as the weight of $I(t)$. Based on the above scheme, the self-awareness of node $i$ at time $t$ can be expressed as

$$
\alpha_{i}(t)=1-(1-\alpha)^{m_{i}+\eta I(t)+1} .
$$

A S-state node with self-awareness $\alpha_{i}(t)$ at time $t$ will take protective measures, such as wearing masks and washing hands, which will affect the transmission probability of the disease. Thus, the actual infection rate of $i$ is

$$
\beta_{i}=\left(1-\alpha_{i}\right) \beta .
$$

A larger value of $\alpha_{i}$ means a smaller probability of being infected.

In addition, an exposed node becomes infected at rate $\delta$ at each time step. At the same time, each I-state node $i$ will recover with a recovery rate $\mu_{i}$, which is assumed to be dependent on the resources received from outside [34, 39]. Therefore, the recovery rate of node $i$ is assumed to be proportional to its resource quantity $\omega_{i}(t)$ in this paper, and is defined as

$$
\mu_{i}(t)=1-(1-\mu)^{\varepsilon \omega_{i}(t)+1},
$$

where the parameters $\mu$ and $\varepsilon \in[0,1]$ represent the basic recovery rate and the resource utilization rate, [40] respectively, since in real life, the phenomenon of resource waste is inevitable in medical and other service systems [41]. Besides, the fraction of susceptible, exposed, infected, and recovered nodes at time $t$ is denoted by $s(t), e(t), i(t)$, and $r(t)$, respectively. Note that we also denoted the final infected density in the dynamical system as $\rho$, which satisfies $\rho \equiv r(\infty)$.

2.2. Self-Awareness-Based Resource Allocation Model. People aware of the disease would change the attitude and behavior of resource donation in suppressing the disease spreading and thus influences the epidemic dynamics. To investigate the impact of awareness-driven individual resource allocation, a resource allocation model is proposed. We assume that each healthy node can generate one unit resource at a time step. Subsequently, the healthy node will donate its resource to help the recovery of the I-state neighbors with probability $q_{i}(t)$, which is determined by the self-awareness $\alpha_{i}(t)$. Intuitively, for self-protection, the higher the level of self-awareness, the lower the probability of resource donation. Consequently, the resource donation probability $q_{i}(t)$ can be expressed as

$$
q_{i}(t)=q_{0}\left(1-\alpha_{i}(t)\right)
$$

where $q_{0}$ is the basic donation probability. Besides, it is assumed that the resources contributed by the healthy nodes will be allocated equally to their infected neighbors at each time step. Combining the donation probability $q_{i}$ and the resource allocation scheme, the amount of resources that each healthy node $i$ allocates to one of its I-state node $j$ at time $t$ is

$$
\omega_{i \rightarrow j}(t)=q_{i}(t) \frac{1}{m_{i}(t)} .
$$

Based on equation (5), the resource quantity of each I-state node $j$ can be expressed as

$$
\begin{aligned}
\omega_{j}(t) & =\sum_{i} a_{i j} h_{i} \omega_{i \longrightarrow j}(t) \\
& =\sum_{i} a_{i j} h_{i} \frac{q_{i}(t)}{m_{i}(t)},
\end{aligned}
$$

where $h_{i}$ is introduced to represent the state of node $i$. When node $i$ is in susceptible state, $h_{i}=1$; otherwise, $h_{i}=0$.

\section{Simulation Results}

In this section, we study systematically the effects of selfawareness on the spreading dynamics by incorporating the allocation of individual resources. First of all, we investigate the coupled awareness-disease dynamics on scale-free networks with fixed degree exponential, as many real-world networks have skewed degree distributions [42-44]. Then, we study the impact of network heterogeneity on the coupled dynamics through extensive Monte Carlo simulations.

In the simulations, we adopt the synchronous updating method [45] to mimic the processes of disease transmission and resource allocation on the complex networks. Specifically, the processes update as follows [45]: during a time interval $[t, t+\Delta t]$, each susceptible node is infected by one of its infected neighbors with probability $\beta_{i} \Delta(t)$ and subsequently, it changes to the exposed state. The actual infection rate $\beta_{i}$ is expressed as [46]

$$
\beta_{i}(t)=\lim _{\Delta t \longrightarrow 0} \frac{P\left(H_{t+\Delta t}^{i}=I \text { infected by } j \mid H_{t}^{i}=S, H_{t}^{j}=I\right)}{\Delta t},
$$

where $H_{t}^{i}$ is denoted as the state of node $i$ at time $t$ and $\left(H_{t+\Delta t}^{i}=I\right.$ infected by $\left.j\right)$ denotes that node $i$ is infected by an I-state neighbor $j$ [47]. At the same time, the E-state nodes becomes I-state ones with probability $\delta \Delta t$, and the I-state nodes change to R-state nodes with probability $\mu_{i}(t) \Delta t$, which is defined as

$$
\mu_{i}(t)=\lim _{\Delta t \rightarrow 0} \frac{P\left(H_{t+\Delta t}^{i}=R \mid H_{t}^{i}=I\right)}{\Delta t} .
$$


The infection rate $\beta_{i}(t)$ and recovery rate $\mu_{i}(t)$ are determined by the number of infected neighbors $m_{i}(t)$, the global confirmed nodes $I(t)$, and the resource quantity $\omega_{i}(t)$ simultaneously.

The process of resource allocation evolves simultaneously with the spread of disease. In synchronous updating, the $\Delta t$ is finite, and the infection and recovery probability of node $i$ is $\beta_{i}=\beta_{i} \Delta t$, and $\mu_{i}=\mu_{i} \Delta t$. According to equations (7) and (8), the transition probability can be expressed as

$$
\beta_{i} \Delta t=P\left(H_{t+\Delta t}^{i}=I \text { infected by } j \mid H_{t}^{i}=S, H_{t}^{j}=I\right),
$$

and the recovery probability can be written as

$$
\mu_{i} \Delta t=P\left(H_{t+\Delta t}^{i}=R \mid H_{t}^{i}=I\right) .
$$

At the end of each time step, the state of all nodes in the network update synchronously. The processes terminate when there are no I-state and E-state nodes in the network.

\subsection{Effects of Awareness-Driven Resource Allocation on the} Spreading Dynamics. To study the coupled dynamics in complex networks, we use the uncorrelated configuration model (UCM) [48] to generate networks with power-law degree distributions $P(k)=\zeta k^{-\gamma}$, where the coefficient is $\zeta=1 / \sum_{k_{\min }}^{k_{\max }} k^{-\gamma}$, as many real-world networks have skewed degree distributions $[43,49]$. The size of the network is set to $N=10000$, and the average degree is $\langle k\rangle=8$. To ensure that there is no degree correlation of the network, the maximum and minimum degrees are set to $k_{\max }=\sqrt{N}$ and $k_{\min }=3$, respectively [50]. We focus on the case of $\gamma=2.4$ in this section. To initiate a spreading process, a fraction $i(0)=$ 0.001 of nodes is selected randomly as seeds and the remaining nodes are in susceptible state.

To numerically determine the size-dependent epidemic threshold $\beta_{c}$, we employ the variability measure [51], which has been demonstrated to be effective in identifying the epidemic threshold of SIR model [52]:

$$
\Delta=\frac{\sqrt{\left\langle\rho^{2}\right\rangle-\langle\rho\rangle^{2}}}{\langle\rho\rangle},
$$

where the operator $\langle\ldots\rangle$ represents the ensemble average over all realizations. We can identify the epidemic threshold $\beta_{c}$, at which the variability $\Delta$ exhibits a peak.

Figure 1(a) displays the final fraction of infected nodes $\rho$ as a function of basic transmission rate $\beta$ for different values of instinctive awareness $\alpha$. Figure 1(b) shows the plots of variability measure $\Delta$ vs. $\beta$. The initial fraction of infected nodes is set to be $i(0)=0.001$. We observe that the threshold $\beta_{c}$ increases with the increase of $\alpha$, see the peaks of $\Delta$ in Figure 1(b). In addition, when the basic infection rate is fixed, e.g., $\beta=0.2$, the value of $\rho$ decreases with the increase of $\alpha$, as shown in Figure 1(a). The results suggest that the more self-protective people are during the outbreak of an epidemic, the more effectively the disease can be controlled.

Next, we study systematically the effects of self-awareness $\alpha$ and basic transmission rate $\beta$ on the spreading dynamics by presenting the full phase diagram in parameter plane $(\alpha-\beta)$ in Figure 2(a). Colors in Figure 2(a) encode the value of $\rho$. The white circles connected by segments identify the epidemic thresholds $\beta_{c}$ at different values of $\alpha$, which are obtained by the method of variability measure presented in equation (11). We observe that the overall the epidemic threshold $\beta_{c}$ increases monotonously with the increase of $\alpha$. Besides, when $\alpha$ is relatively small (in phase I), the value of $\beta_{c}$ increases slowly, and the final fraction of infected nodes $\rho$ increases abruptly with $\beta$ at a fixed value of $\alpha$. And then, when the $\alpha$ grows larger (in phase II), the final infected density $\rho$ increases slowly with $\beta$ at a fixed $\alpha$. Finally, when $\alpha$ is large enough (in phase III), the disease can not break out any more.

To identify the critical points of $\alpha$, we calculate the change rate of $\beta_{c}$, which is defined as

$$
v_{r} \equiv \frac{\mathrm{d} \beta_{c}}{\mathrm{~d} \alpha}=\lim _{\Delta \alpha \longrightarrow 0} \frac{\beta_{c}(\alpha+\Delta \alpha)-\beta_{c}(\alpha)}{\Delta \alpha},
$$

where $\Delta \alpha$ is the increment in $\alpha$ and is set to be $\Delta \alpha=0.01$. By performing extensive Monte Carlo simulations, we calculate the value of $v_{r}$ at each point of $\alpha$, and identify the two critical points, namely, the first point $\alpha_{c}^{I} \approx 0.64$, at which the value of $v_{r}$ exceeds an threshold value that is preset to 0.1 , and the second point $\alpha_{c}^{\mathrm{II}}$, at which $v_{r}$ reaches maximum value. Figure 2(b) shows the plot of $v_{r}$ as a function of $\alpha$. We can observe that the parameter space is separated by the two critical values into three phases, which is in accordance with the three phases marked by phase I, phase II, and phase III in Figure 2(a), respectively.

The above results suggest that during the outbreak of an epidemic, as long as the populations maintain sufficient awareness of self-protection, say $\alpha>\alpha_{c}^{\mathrm{I}}$, and take effective protective measures, such as conserving their resources to reduce waste of resources and reduce exposure, the disease can be suppressed effectively.

Next, we qualitatively explain the above conclusions by studying the time evolution of several important dynamical parameters at three typical values of $\alpha$ located in $\alpha<\alpha_{c}^{\mathrm{I}}$, $\alpha_{c}^{\mathrm{I}} \leq \alpha<\alpha_{c}^{\mathrm{II}}$ and $\alpha \geq \alpha_{c}^{\mathrm{II}}$, respectively. Note that, in order to study the evolution of the dynamic parameters for nodes with large degrees and small degrees, respectively, we define the nodes with degrees larger than 30 as the hub nodes, and the remaining nodes as small-degree nodes. Subsequently, we define the average awareness, transmission rate, recovery rate, and probability of resource donation as $\left\langle\alpha_{x}\right\rangle,\left\langle\beta_{x}\right\rangle$, $\left\langle\mu_{x}\right\rangle$, and $\left\langle q_{x}\right\rangle$, respectively, where $x \in(h, s)$. For the hub nodes, $x=h$, and for the small-degree nodes, $x=s$.

First of all, we investigate the time evolution of the dynamical parameters in $\alpha<\alpha_{c}^{\mathrm{I}}$, as shown in Figure 3 . Without loss of generality, the instinctive self-awareness is set to be $\alpha=0.1$. Figure 3(a) displays the evolution of the dynamic parameters for the hub nodes. We find that when $\alpha=0.1$, nodes in the network have a relatively small instinctive self-awareness and a large probability of resource donation to support the recovery of the I-state nodes in the initial time. Therefore, we can learn from equation (3) that the recovery rate of the nodes is large. Consequently, it has a relatively small value of the effective infection rate $\beta_{e}$, which 


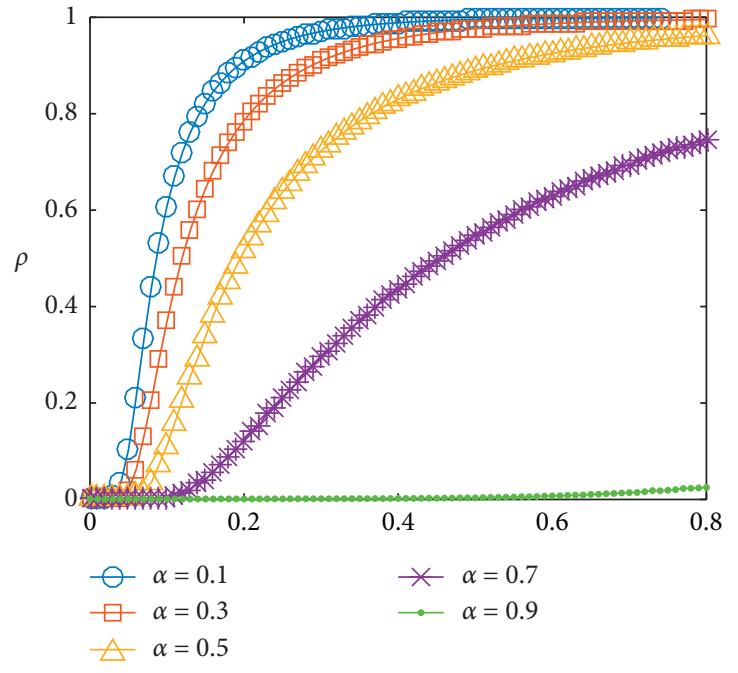

(a)

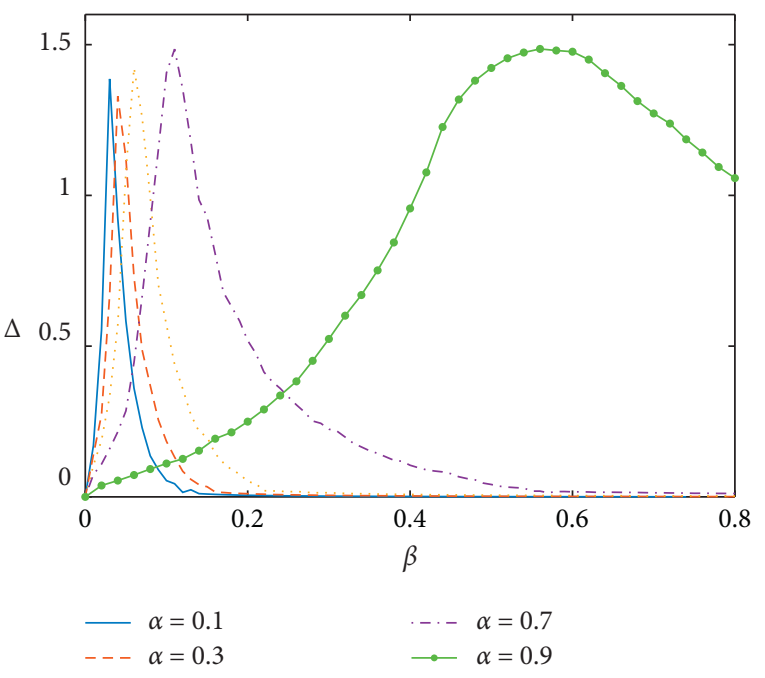

(b)

Figure 1: Effects of awareness-driven individual resource allocation on the spreading dynamics in scale-free networks. (a) The final infected density $\rho$ as a function of basic transmission rate $\beta$ for five typical values of instinctive self-awareness $\alpha$. (b) Plots of variability measure $\Delta$ vs. $\beta$. The other parameters are set to be $\delta=0.5, \eta=0.001, \varepsilon=0.6, \mu=0.1$, and $q_{0}=0.8$, respectively. Data are obtained by averaging over $10^{2} \times 10^{2}$ independent realizations on $10^{2}$ networks.

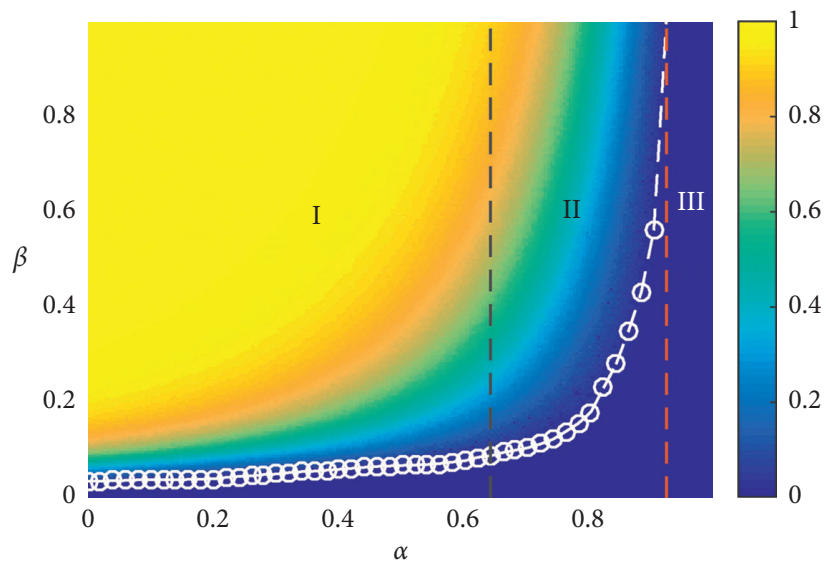

(a)

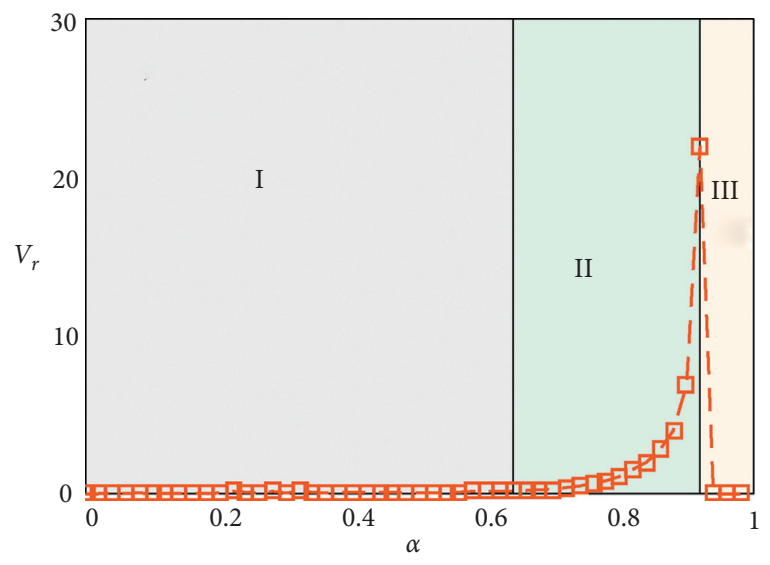

(b)

Figure 2: Dependence of $\rho$ on parameters $\alpha$ and $\beta$. (a) The phase diagram in parameter plane $(\alpha-\beta)$. Colors-coded values $\rho$ obtained from Monte Carlo simulations. The epidemic threshold $\beta_{c}$ is represented by the white circles. Phases I, II, and III are separated by two critical values $\alpha_{c}^{\mathrm{I}} \approx 0.64$ and $\alpha_{c}^{\mathrm{II}} \approx 0.92$, which are marked by the black segment and red segment, respectively. (b) The change rate of the epidemic threshold $v_{r}$ as a function of $v_{r} \alpha$. Phases I, II, and III are separated by $\alpha_{c}^{\mathrm{I}}$ and $\alpha_{c}^{\mathrm{II}}$. The other parameters are set to be $\delta=0.5, \eta=0.001, \varepsilon=0.6$, $\mu=0.1$, and $q_{0}=0.8$, respectively.

is defined as $\beta_{e}=\beta / \mu$ [53]. Thus, we can observe from Figure $3(\mathrm{c})$ that the disease propagates slowly in the initial stage (about $t<5$ ). The slow growth of the infected nodes leads to a weak awareness of the disease for the susceptible nodes. Thus, at the early stage, i.e., $t<5$, there is a slow growth in the self-awareness and a slight decrease in the resource donation probability for all nodes in the network, as shown in Figures 3(a) and 3(b). Whereas, after the early stage, i.e., $t>10$, there is a rapid increase in the exposed nodes $e(t)$ and infected nodes $i(t)$ (see Figure 3(c)). The surge in the number of infected nodes induces a rapid improvement of the self-awareness, which makes a rapid increase in $\left\langle\alpha_{h}\right\rangle$, as shown in Figure 3(a). However, the value of $\left\langle\alpha_{s}\right\rangle$ and $\left\langle q_{s}\right\rangle$ changes slowly, as shown in Figure 3(b). The above phenomena can be explained as follows: the propagation process of the epidemic exhibits hierarchical feature in the network [54], namely, the hubs are likely to be infected firstly, and then they transmits the disease to the intermediate nodes; finally, the small-degree nodes are infected. As a result, as more and more hub nodes become 


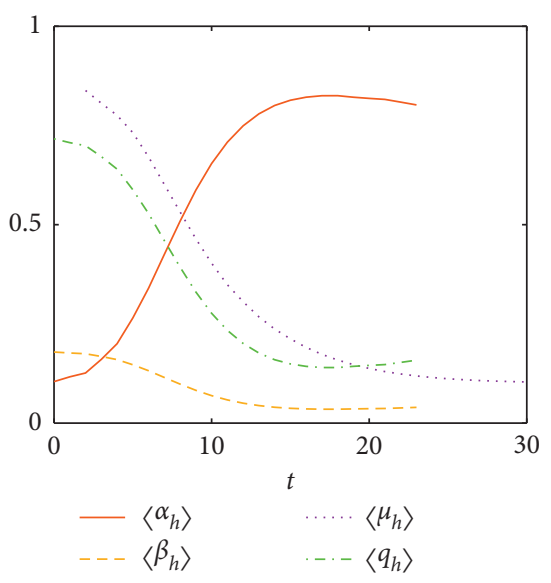

(a)

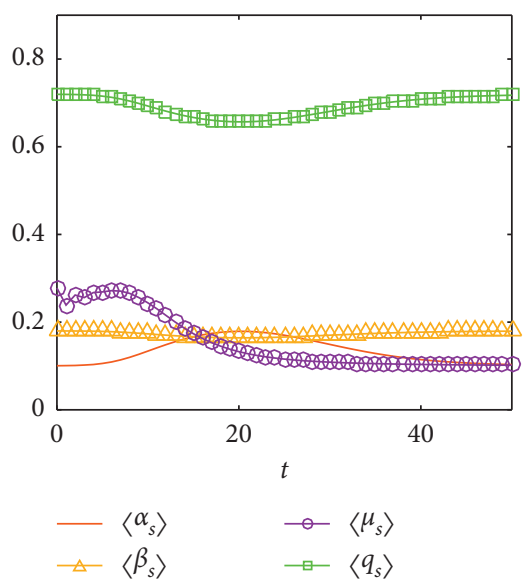

(b)

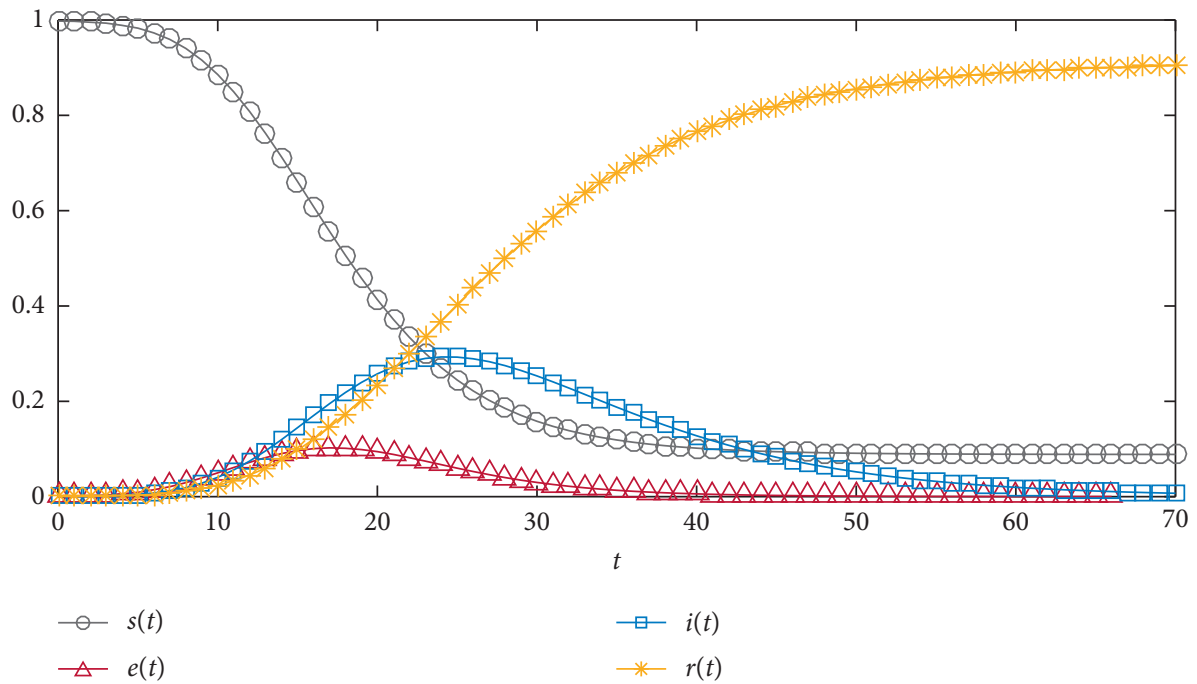

(c)

FIgURE 3: (a) Temporal evolution of average values of awareness $\left\langle\alpha_{h}\right\rangle$ (red line), transmission rate $\left\langle\beta_{h}\right\rangle$ (yellow segment), recovery rate $\left\langle\mu_{h}\right\rangle$ (purple dots), and probability of resource donation $\left\langle q_{h}\right\rangle$ for hub nodes. (b) The evolution of average values of awareness $\left\langle\alpha_{s}\right\rangle$ (red line), infection rate $\left\langle\beta_{s}\right\rangle$ (yellow triangles), recovery rate $\left\langle\mu_{s}\right\rangle$ (purple circles), and probability of resource donation $\left\langle q_{s}\right\rangle$ (green squares) for nodes with small degree. (c) The evolution of the fraction of the nodes in susceptible $s(t)$, exposed $e(t)$, infected $i(t)$, and recovered $r(t)$ states, respectively. The instinctive self-awareness is $\alpha=0.1$, and the basic infection rate is $\beta=0.2$. The other parameters are the same as those in Figure 2. The results of the simulations are obtained by averaging over $10^{2} \times 10^{2}$ independent realizations on $10^{2}$ networks.

infected, the disease transmits rapidly in a hub node-centered area, and the value of $e(t)$ and $i(t)$ increases rapidly. Consequently, there is an abrupt increase in $\left\langle\alpha_{h}\right\rangle$ and a rapid decrease in the resource donation probability $\left\langle q_{h}\right\rangle$ in this stage, which induces a decrease in both the transmission rate $\left\langle\beta_{h}\right\rangle$ and simultaneously, the recovery rate $\left\langle\mu_{h}\right\rangle$ as the shortage of treatment resources. We can observe that the effective infection rate $\beta_{e}$ increases in this stage as the value of $\left\langle\beta_{h}\right\rangle$ drops more rapidly than $\left\langle\beta_{h}\right\rangle$. Consequently, the disease continues spreading in the network, and finally a large fraction of nodes are infected by the disease.

As for the small-degree nodes, as there are fewer connections with the outside, they have less information about the status of the epidemic and a lower self-protection awareness compared with the hub nodes in the early stage. Thus, the values of $\left\langle\alpha_{s}\right\rangle$ and $\left\langle q_{s}\right\rangle$ change slowly in this stage
(Figure 3(b)). However, in the later stage, due to the protective measures adopted by hub nodes, the transmission of disease from hub nodes to small-degree nodes is basically blocked. Thus, there is a slight decrease in $\left\langle\alpha_{s}\right\rangle$ and a small increase in $\left\langle q_{s}\right\rangle$ in this stage.

Based on the above description, we can reasonably explain the phenomenon in phase I in Figure 2 that the disease increases abruptly in this phase.

Secondly, we study the time evolution of the dynamical parameters in $\alpha_{c}^{\mathrm{I}} \leq \alpha<\alpha_{c}^{\mathrm{II}}$ in Figure 4 . We find that, when $\alpha=0.7$, nodes in the network have a larger instinctive selfawareness compared with the case of $\alpha=0.1$. In this case, due to the hierarchical nature of disease transmission, a small number of global and local infected nodes $\left(I_{i}\right.$ and $\left.m_{i}\right)$ will lead to a high vigilance of the hub nodes. Therefore, the value of $\left\langle\alpha_{h}\right\rangle$ increases abruptly before time $t \approx 50$, as shown 


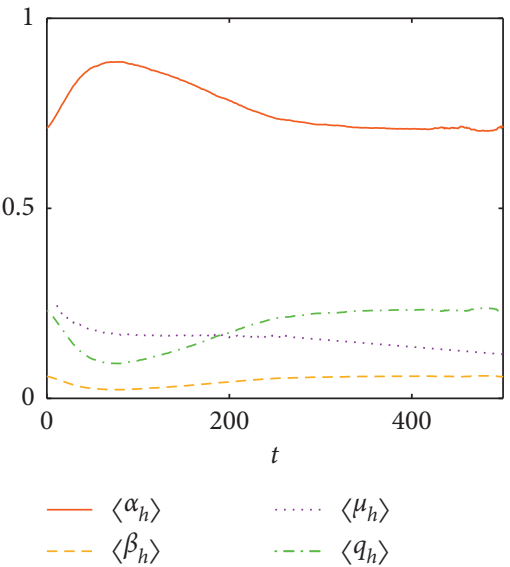

(a)

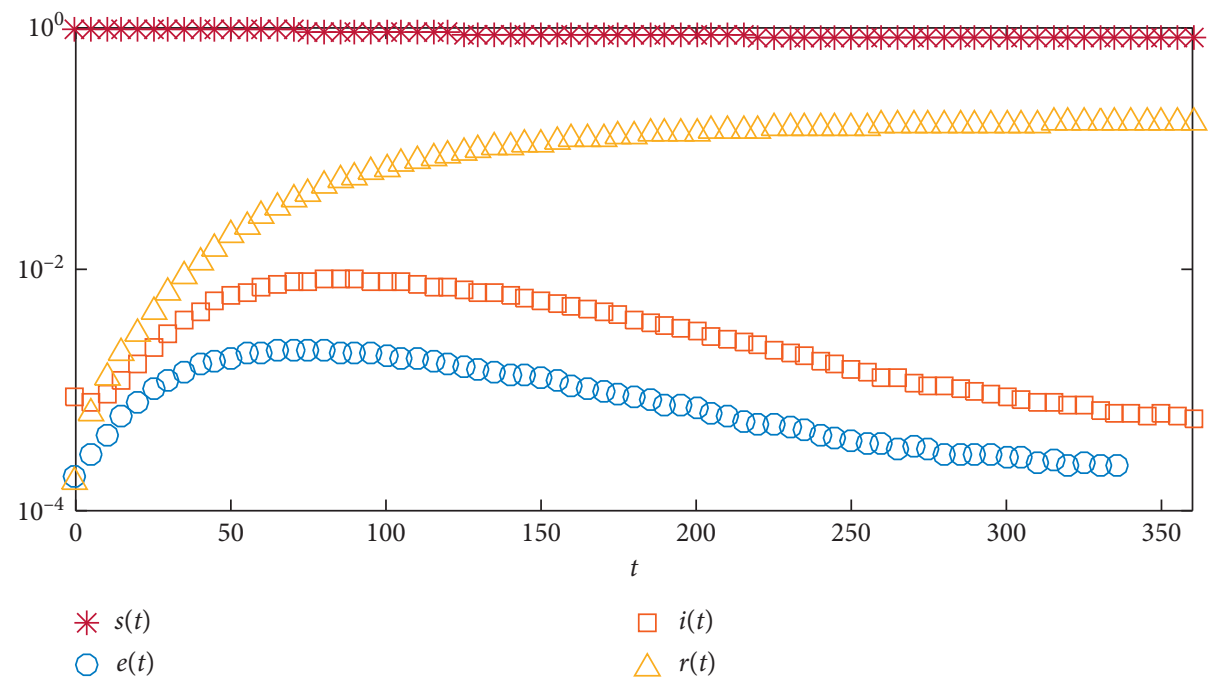

(c)

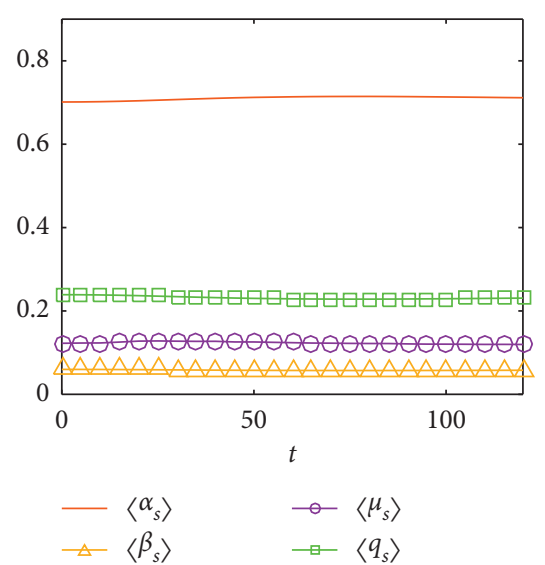

(b)

FiguRe 4: (a) Temporal evolution of the average values of awareness $\left\langle\alpha_{h}\right\rangle$ (red line), infection rate $\left\langle\beta_{h}\right\rangle$ (yellow segment), recovery rate $\left\langle\mu_{h}\right\rangle$ (purple dots), and probability of resource donation $\left\langle q_{h}\right\rangle$ for hub nodes. (b) The evolution of the average values of awareness $\left\langle\alpha_{s}\right\rangle$ (red line), infection rate $\left\langle\beta_{s}\right\rangle$ (yellow triangles), recovery rate $\left\langle\mu_{s}\right\rangle$ (purple circles), and probability of resource donation $\left\langle q_{s}\right\rangle$ (green squares) for nodes with small degree. (c) The evolution of fraction of the nodes in susceptible $s(t)$, exposed $e(t)$, infected $i(t)$, and recovered $r(t)$ state, respectively. The instinctive self-awareness is $\alpha=0.7$ and the basic infection rate is $\beta=0.2$.

in Figure 4(a), which leads to a rapid decrease of resource donation probability $\left\langle q_{h}\right\rangle$ and a reduction in the transmission rate $\left\langle\beta_{h}\right\rangle$. In this condition, the hub nodes are protected from been infected, which can also stop the reinfection of the hub nodes, as they can connect directly to each other or via a few small nodes $[55,56]$. Further, the reduction of the resources will lead to a decrease in recovery rate $\left\langle\mu_{h}\right\rangle$, as shown in Figure $4(\mathrm{a})$. Besides, the effective infection rate $\beta_{e}$ keeps a relatively small value in the initial stage, where the fraction of infected and exposed nodes $i(t)$ and $e(t)$ increases slowly. It indicates that the epidemic spreading is suppressed effectively compared with the case of $\alpha=0.1$. After that, with the gradual recovery of the I-state nodes, there are fewer and fewer I-state neighbors around the hub nodes, which makes them gradually relax their vigilance against the disease and lead to the gradual decline of $\left\langle\alpha_{h}\right\rangle$. This phenomenon is fully consistent with the case that, in real scenarios, the slowdown in the epidemic spreading will lead to increased daily contact and economic recovery activities $[57,58]$. As shown in Figure 4(a), the value of $\left\langle\alpha_{h}\right\rangle$ decreases after $t \approx 50$, which is accompanied by the increase of $\left\langle q_{h}\right\rangle$. The decrease of $\left\langle\alpha_{h}\right\rangle$ leads to a rise in the effective infection rate $\left\langle\beta_{e}\right\rangle$ (see the gap between $\left\langle\beta_{h}\right\rangle$ and $\left\langle\mu_{h}\right\rangle$ ), which will lead to a rise in the infected nodes, whereas the increase of infected nodes can immediately lead to the enhancement of self-protection awareness of hub nodes, as they have a high instinctive self-awareness, which in turn suppresses the spread of disease. Consequently, we can learn that there is a dynamic balance between the spread of disease and individuals' self-protection awareness after $t \approx 100$ (Figure $4(a)$ ). Thus, there is a lower value of final infected density $\rho$ compared to the case in phase I, and the disease can be suppressed to a certain extent. 
Based on the above description, we can reasonably explain the reason why the disease increases slowly in phase II of Figure 2. Similarly, we can also explain the phenomena in phase III of Figure 2. Specifically, the hub nodes are very sensitive to the spread of disease as they all have a large value of instinctive self-awareness. Consequently, a small increase in the values of $m_{i}(t)$ and $I(t)$ will lead to the rapid increase in $\left\langle\alpha_{h}\right\rangle$ at the very early stage of spreading process. Therefore, the hub nodes are effectively protected from being infected and the disease is well suppressed at the beginning of the transmission process. Therefore, the disease will not breakout any more.

\subsection{Effects of Network Heterogeneity on the Coupled Disease-} Awareness Dynamics. In this section, we study the effects of network heterogeneity on the coupled dynamics of selfawareness and epidemic spreading. Since the network heterogeneity decreases with the increase of the power exponent $\gamma$ [55], we construct the networks with different heterogeneity by changing the degree exponent based on the UCM model.

First of all, we study the value of $\rho$ as a function of $\beta$ in networks with different degree exponents. In Figure 5(a), the curves for the four typical values of $\gamma$ are displayed. Interestingly, we find that the network heterogeneity plays the role of "double-edged sword" in that they can either suppress or promote the epidemic spreading. Specifically, we find that the curves of the infected density intersect at a fixed basic infection rate about $\beta^{*} \approx 0.13$ and dynamical processes are separated into two phases. In phase I, i.e., HTML transalation failed, the basic infection rate is small, the final infected density $\rho$ increases with the increase of network heterogeneity at a fixed value of $\beta$, and the epidemic threshold $\beta_{c}$ increases with $\gamma$. While, in phase II, i.e., $\beta>\beta^{*}$, the value of $\beta$ is large, the value of $\rho$ decreases with the increase of network heterogeneity. The results indicate that when the basic infection rate $\beta$ is small, the network heterogeneity promotes disease spreading, which is consistent with the results in the classical epidemiological model [59]. While, when there is a large value of $\beta$, e.g., $\beta=0.2$, it suppresses the outbreak of disease.

To illustrate the conclusions more clearly, we plot the value of $\rho$ as a function of $\gamma$ at two typical values of $\beta$ in the two regions $\beta<\beta^{*}$ and $\beta>\beta^{*}$, respectively, in Figure 5(b). Without loss of generality, we select $\beta=0.1$ in phase I and $\beta=0.2$ in phase II, respectively. Through extensive simulations, we find that when $\beta=0.1$, the value of $\rho$ increases monotonously with $\gamma$; in contrast, it decreases with $\gamma$ when $\beta=0.2$.

Further, we study the relationship between the epidemic threshold $\beta_{c}$ and the self-awareness $\alpha$ for different values of $\gamma$. Figure 6 displays the value of $\beta_{c}$ as a function of $\alpha$. We find that the value of $\beta_{c}$ increases monotonously with the increase of $\alpha$ for each value of $\gamma$. Specially, the value of $\beta_{c}$ increases abruptly when $\alpha>\alpha_{c}^{\mathrm{I}}$ defined in Figure 2. When $\alpha$ is fixed, the value of $\beta_{c}$ increases $\gamma$.

We can use the same theory as in the previous section to qualitatively explain the "double-edged sword" phenomenon. Specifically, in a network with strong heterogeneity, the vast majority of nodes have small degrees and only a few nodes have a large number of connections. Due to the hierarchical character of the disease transmission, the disease is likely to spread rapidly in a hub node-centered area. Thus, when the basic infection rate $\beta$ is large, e.g., $\beta=0.2$, the number of infected nodes in the local area increases rapidly, which induces to a rapid increase in the selfawareness of the hub nodes $\left\langle\alpha_{h}\right\rangle$. Subsequently, the infection rate of the hub nodes $\left\langle\beta_{h}\right\rangle$ drops significantly, which reduces the infection between hub nodes and the smalldegree nodes. Therefore, the stronger the network heterogeneity, the more the disease transmission can be suppressed. On the contrary, when the transmission rate is small, e.g., $\beta=0.1$, this inhibition to disease transmission will disappear. In this case, the structural heterogeneity will promote the spread of the disease.

At last, to further investigate the "double-edged sword" effects of network heterogeneity on the coupling dynamics, we study the relationship between the final fraction of infected node $\rho$ and self-awareness $\alpha$ on networks with different degree exponents when there is as relatively large basic infection rate, e.g., $\beta=0.2$. Interestingly, once again, we find the "double-edged sword" effect of network heterogeneity on dynamic properties. As shown in Figure 7(a), the value of $\rho$ decreases with $\alpha$ and the curves intersect at a critical value $\alpha^{*}$ that is equal to the first critical point in Figure 2. When $\alpha<\alpha^{*}$ (in phase I), the value of $\rho$ increases with $\gamma$ (see the curve for $\alpha=0.5$ in Figure 7(b)), which indicates that the network heterogeneity inhibits the spread of disease. The above result is in accordance with that in phase II of Figure 5(a). On the contrary, when $\alpha>\alpha^{*}$, the value of $\rho$ decreases with $\gamma$ (see the curve for $\alpha=0.7$ in Figure 7(b)), which indicates that the network heterogeneity promotes the spread of disease in this phase.

Next, we continue explaining the reason why the network heterogeneity promotes the spread of disease in phase II of Figure 7(a). Specifically, when $\alpha>\alpha^{*}$, there is a strong instinctive self-awareness for all nodes in networks. When there is strong degree heterogeneity of a network, e.g., $\gamma=2.1$, the vast majority of nodes have small degrees and only a few nodes have a large number of connections. Due to the hierarchical character of the disease transmission, the disease is likely to spread rapidly in a hub node-centered area, which means that the value of $\left\langle\alpha_{h}\right\rangle$ increases abruptly in the early stage of an epidemic according to equation (1). Thus, there is a small value of $\left\langle\beta_{h}\right\rangle$. Consequently, the hubs are protected effectively in this condition. However, as the small-degree nodes that make up the vast majority of the network have few connections with other nodes, the number of their infected neighbors $m_{i}$ is very small. Therefore, there is a relatively small value of $\left\langle\alpha_{s}\right\rangle$. Consequently, the infection rate $\left\langle\beta_{s}\right\rangle$ of the small-degree nodes is relatively large, which leads to a large probability to be infected for these nodes. On the contrary, in the network with a relatively homogeneous degree distribution, e.g., $\gamma=4.0$, the proportion of nodes with large degree is larger than that of networks with strong degree heterogeneity. Thus, a larger proportion of nodes have a larger value of $\left\langle\alpha_{h}\right\rangle$ in these 


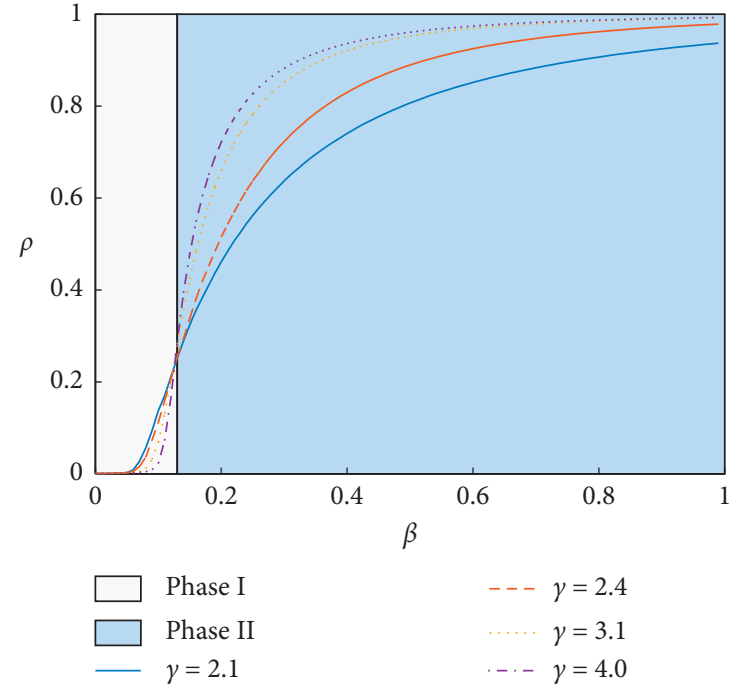

(a)

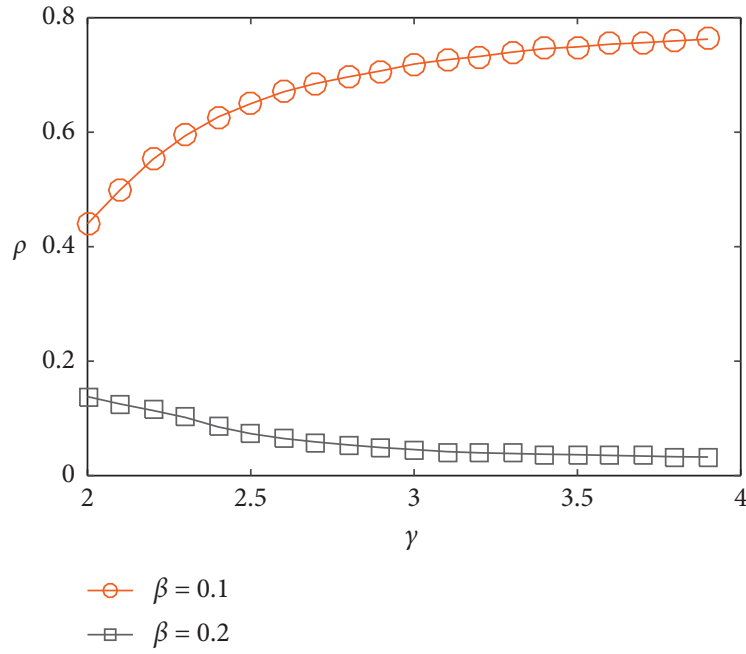

(b)

Figure 5: Effects of network heterogeneity on the coupled dynamics. (a) The final infected density $\rho$ as a function of $\beta$ when degree exponents $\gamma=2.1$ (blue line), $\gamma=2.4$ (red line segment), $\gamma=3.1$ (yellow dots), and $\gamma=4.0$ (purple dotted line), respectively. The two regions marked by phase I (filled by gray) and phase II (filled by sky blue) are separated by the critical value $\beta^{*} \approx 0.13$. (b) The value of $\rho$ as a function of $\gamma$ for $\beta=0.1$ (red circles) and $\beta=0.2$ (gray squares), respectively. The instinctive self-awareness is fixed as $\alpha=0.5$. The other parameters are the same as those in Figure 1.

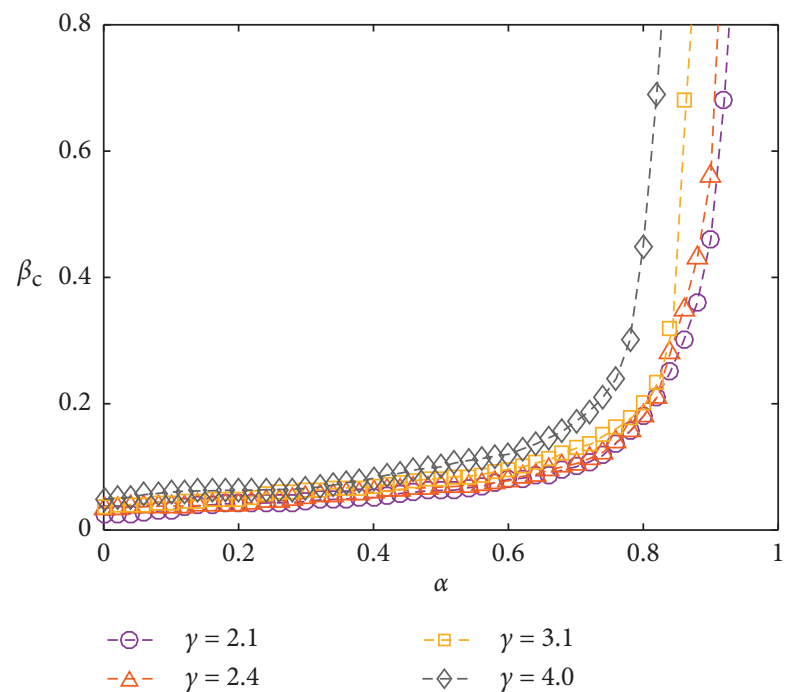

FIGURE 6: The relationship between the epidemic threshold $\beta_{c}$ and the instinctive self-awareness $\alpha$ when the degree exponents are set at $\gamma=2.1$ (purple circles), $\gamma=2.4$ (red triangles), $\gamma=3.1$ (yellow squares,) and $\gamma=4.0$ (gray rhombuses), respectively. The other parameters are the same as those in Figure 1. Data are obtained by averaging over $10^{2} \times 10^{2}$ independent realizations on $10^{2}$ networks.

networks, and the disease can be suppressed under this condition.

\section{Discussion}

The self-protection awareness of the individuals can influence their behavior response to the epidemics, such as reducing contacts with others and large public gatherings.
More importantly, it will also affect the behavior of individual resource allocation that is of critical importance in controlling the outbreak of an epidemic. To investigate the effects of the self-awareness-driven individual resource support on the epidemic dynamics, we have proposed a resource-based susceptible-exposed-infected-recovered ( $\mathrm{r}$ SEIR) epidemiological model and a self-awareness-based resource allocation model, respectively. In the models, the self-awareness of the individuals is assumed to come from both local communities and global mass media, which is measured by the number of infected neighbors and global infected nodes.

Firstly, we have studied the effects of the awarenessdriven individual resource allocation on the spreading dynamics in sale-free networks with fixed degree exponent. Through extensive Monte Carlo simulations, we have found that the final infected density $\rho$ decreases with the increase of the instinctive self-awareness $\alpha$. More importantly, there are two critical values $\alpha_{c}^{\mathrm{I}}$ and $\alpha_{c}^{\mathrm{II}}$ that separate the parameter space into three phases. In phase I, i.e., $\alpha<\alpha_{c}^{\mathrm{I}}$, the final infected density $\rho$ increases abruptly with the basic infection rate $\beta$, which implies that the disease can not be suppressed effectively. In phase II, i.e., $\alpha_{c}^{\mathrm{I}} \leq \alpha<\alpha_{c}^{\mathrm{II}}$, the value of $\rho$ grows gently with $\beta$, which means that the disease can be controlled to a certain extent. At last, in phase III, i.e., $\alpha \geq \alpha_{c}^{\mathrm{II}}$, the disease does not break out in the network and the value of $\rho$ remains a very small value. Combining the study of the time evolution of dynamical parameters, we have qualitatively explained the corresponding phenomena. The results suggest that the disease can be effectively controlled as long as we maintain a proper level of self-protection awareness.

Next, we have investigated the effects of network heterogeneity on the coupled dynamics. Through extensive 


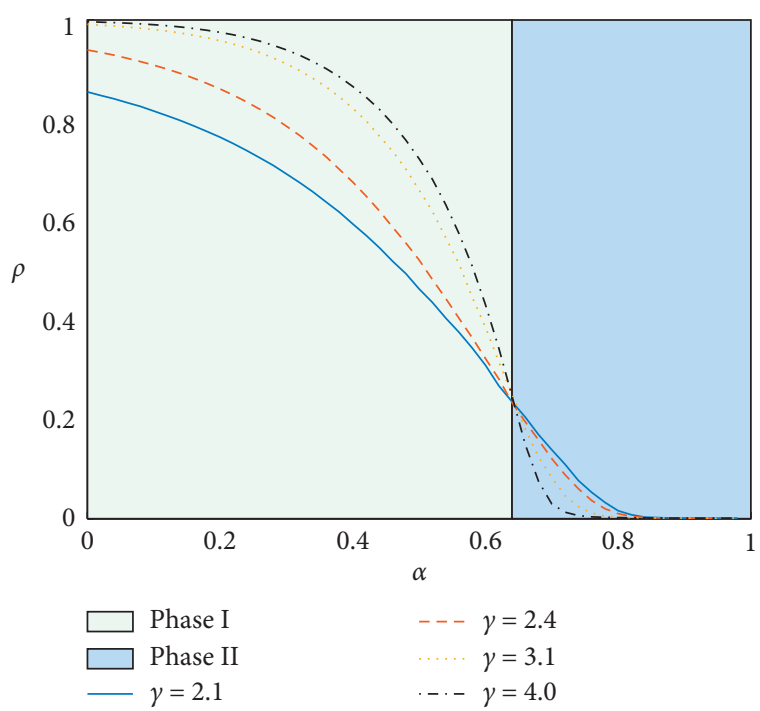

(a)

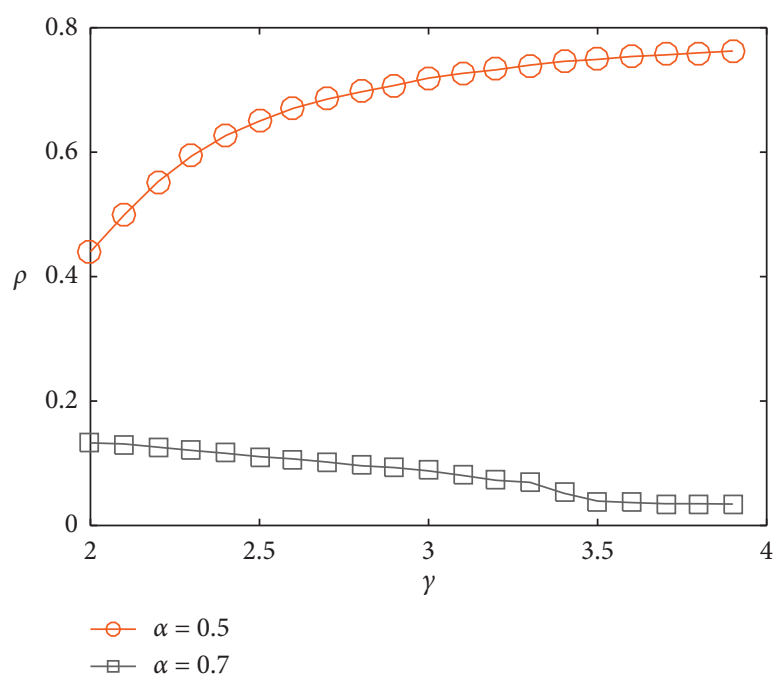

(b)

Figure 7: Effects of self-awareness and network structure on the spreading dynamics. (a) Plots of $\rho$ vs. $\alpha$ when $\gamma=2.1$ (blue line), $\gamma=2.4$ (red line segment), $\gamma=3.1$ (yellow dots), and $\gamma=4.0$ (black dotted line), respectively. The two regions marked by phase I (filled by gray) and phase II (filled by sky blue) are separated by the critical value $\alpha^{*} \approx 0.64$. (b) The value of $\rho$ as a function of $\gamma$ when $\alpha=0.5$ (red circles) and $\alpha=0.7$ (black squares), respectively. The basic transmission rate is set at $\beta=0.2$, and the other parameters are the same as those in Figure 1 .

simulations, we have found that the network heterogeneity has "double-edged sword" effect on the coupling dynamics. Specifically, we have found that there is a critical value of basic infection rate $\beta^{*}$, when $\beta<\beta^{*}$, the network heterogeneity promotes the spread of disease. On the contrary, when $\beta>\beta^{*}$, the network heterogeneity inhibits the spread of disease. To further investigate "double-edged sword" effects of network heterogeneity on the coupling dynamics, we have studied the relationship between $\rho$ and $\alpha$ on networks with different degree exponents when there is as relatively large basic infection rate. Through extensive simulations, we have found the instinctive self-awareness denoted as $\alpha^{*}$, which is in accordance with $\alpha_{c}^{\mathrm{I}}$. When $\alpha<\alpha^{*}$, the network heterogeneity inhibits the spread of disease. On the contrary, when $\alpha>\alpha^{*}$, the network heterogeneity promotes the spread of disease.

Our findings make a substantial contribution to understanding the effects of people's awareness of self-protection on the spreading dynamics during a pandemic, which will be of practical significance in controlling the outbreak of infectious diseases, especially in the context of the COVID-19 pandemic. The results obtained in this paper can guide people to maintain the right self-awareness and behavior during an outbreak and also have a direct application in the development of strategies to suppress the spread of the disease.

\section{Data Availability}

The data used to support the findings of this study are available from the corresponding author upon request.

\section{Conflicts of Interest}

The authors declare that they have no conflicts of interest regarding this paper.

\section{Acknowledgments}

This work was supported by the Fundamental Research Funds for the Central Universities (nos. JBK190972, JBK171113, and JBK170505), National Natural Science Foundation of China (nos. 71671141 and 71873108), the 2020 school-level scientific research project of Aba Teachers University (no. ASA20-01), and the Financial Intelligence and Financial Engineering Key Lab of Sichuan Province.

\section{References}

[1] A. Trilla, G. Trilla, and C. Daer, "The 1918 "Spanish flu" in Spain," Clinical Infectious Diseases, vol. 47, no. 5, pp. 668-673, 2008.

[2] K. H. Chan, P. H. Li, S. Y. Tan, Q. Chang, and J. P. Xie, "Epidemiology and cause of severe acute respiratory syndrome (SARS) in guangdong, people's Republic of China, in february, 2003," The Lancet, vol. 362, no. 9393, pp. 1353-1358, 2003.

[3] M. P. Girard, J. S. Tam, O. M. Assossou, and M. P. Kieny, “The 2009 a (H1N1) influenza virus pandemic: a review," Vaccine, vol. 28, no. 31, pp. 4895-4902, 2010.

[4] WHO, Coronavirus Disease 2019 (Covid-19) Situation Report-209, WHO, Geneva, Switzerland, 2020.

[5] Z. Wang, M. A. Andrews, Z.-X. Wu, L. Wang, and C. T. Bauch, "Coupled disease-behavior dynamics on complex networks: a review," Physics of Life Reviews, vol. 15, pp. 1-29, 2015.

[6] C. Liu and Z.-K. Zhang, "Information spreading on dynamic social networks," Communications in Nonlinear Science and Numerical Simulation, vol. 19, no. 4, pp. 896-904, 2014.

[7] S. Funk, E. Gilad, C. Watkins, and V. A. A. Jansen, "The spread of awareness and its impact on epidemic outbreaks," Proceedings of the National Academy of Sciences, vol. 106, no. 16, pp. 6872-6877, 2009. 
[8] W. Wang, H. E. Stanley, and L. A. Braunstein, "Effects of timedelays in the dynamics of social contagions," New Journal of Physics, vol. 20, no. 1, Article ID 013034, 2018.

[9] J. J. Van Bavel, K. Baicker, P. S. Boggio et al., "Using social and behavioural science to support covid-19 pandemic response," Nature Human Behaviour, vol. 4, no. 5, pp. 460-471, 2020.

[10] H. Peng, W. Peng, D. Zhao, and W. Wang, "Impact of the heterogeneity of adoption thresholds on behavior spreading in complex networks," Applied Mathematics and Computation, vol. 386, Article ID 125504, 2020.

[11] R. Li, W. Wang, and Z. Di, "Effects of human dynamics on epidemic spreading in Côte d'Ivoire," Physica A: Statistical Mechanics and Its Applications, vol. 467, pp. 30-40, 2017.

[12] S. P. Adhikari, S. Meng, Y.-J. Wu et al., "Epidemiology, causes, clinical manifestation and diagnosis, prevention and control of coronavirus disease (covid-19) during the early outbreak period: a scoping review," Infectious Diseases of Poverty, vol. 9, no. 1, pp. 1-12, 2020.

[13] M. U. G. Kraemer, C.-H. Yang, B. Gutierrez et al., "The effect of human mobility and control measures on the covid-19 epidemic in China," Science, vol. 368, no. 6490, pp. 493-497, 2020.

[14] R. Li, P. Richmond, and B. M. Roehner, "Effect of population density on epidemics," Physica A: Statistical Mechanics and Its Applications, vol. 510, pp. 713-724, 2018.

[15] W. Wang, Q.-H. Liu, J. Liang, Y. Hu, and T. Zhou, "Coevolution spreading in complex networks," Physics Reports, vol. 820, pp. 1-51, 2019.

[16] X.-T. Liu, Z.-X. Wu, and L. Zhang, "Impact of committed individuals on vaccination behavior," Physical Review E, vol. 86, no. 5, Article ID 051132, 2012.

[17] H.-F. Zhang, P.-P. Shu, Z. Wang, M. Tang, and M. Small, "Preferential imitation can invalidate targeted subsidy policies on seasonal-influenza diseases," Applied Mathematics and Computation, vol. 294, pp. 332-342, 2017.

[18] K. A. Kabir, K. Kuga, and J. Tanimoto, "The impact of information spreading on epidemic vaccination game dynamics in a heterogeneous complex network- a theoretical approach," Chaos, Solitons \& Fractals, vol. 132, Article ID 109548, 2020.

[19] Q. Guo, Y. Lei, X. Jiang, Y. Ma, G. Huo, and Z. Zheng, "Epidemic spreading with activity-driven awareness diffusion on multiplex network," Chaos: An Interdisciplinary Journal of Nonlinear Science, vol. 26, no. 4, Article ID 043110, 2016.

[20] K. M. A. Kabir, K. Kuga, and J. Tanimoto, "Analysis of SIR epidemic model with information spreading of awareness," Chaos, Solitons \& Fractals, vol. 119, pp. 118-125, 2019.

[21] P. Zhu, X. Wang, S. Li, Y. Guo, and Z. Wang, "Investigation of epidemic spreading process on multiplex networks by incorporating fatal properties," Applied Mathematics and Computation, vol. 359, pp. 512-524, 2019.

[22] Z. Wang, Q. Guo, S. Sun, and C. Xia, "The impact of awareness diffusion on sir-like epidemics in multiplex networks," Applied Mathematics and Computation, vol. 349, pp. 134-147, 2019.

[23] Q. Wu, X. Fu, M. Small, and X.-J. Xu, "The impact of awareness on epidemic spreading in networks," Chaos: An Interdisciplinary Journal of Nonlinear Science, vol. 22, no. 1, Article ID 013101, 2012.

[24] C. Granell, S. Gómez, and A. Arenas, "Dynamical interplay between awareness and epidemic spreading in multiplex networks," Physical Review Letters, vol. 111, no. 12, Article ID 128701, 2013.

[25] X.-X. Zhan, C. Liu, G. Zhou et al., "Coupling dynamics of epidemic spreading and information diffusion on complex networks," Applied Mathematics and Computation, vol. 332, pp. 437-448, 2018.

[26] M. L. Ranney, V. Griffeth, and A. K. Jha, "Critical supply shortages - the need for ventilators and personal protective equipment during the covid-19 pandemic," New England Journal of Medicine, vol. 382, no. 18, p. e41, 2020.

[27] C. Nowzari, V. M. Preciado, and G. J. Pappas, "Optimal resource allocation for control of networked epidemic models," IEEE Transactions on Control of Network Systems, vol. 4, no. 2, pp. 159-169, 2015.

[28] A. Y. Lokhov and D. Saad, "Optimal deployment of resources for maximizing impact in spreading processes," Proceedings of the National Academy of Sciences, vol. 114, no. 39, pp. E8138-E8146, 2017.

[29] H. Chen, G. Li, H. Zhang, and Z. Hou, "Optimal allocation of resources for suppressing epidemic spreading on networks," Physical Review E, vol. 96, no. 1, Article ID 012321, 2017.

[30] S. Li, D. Zhao, X. Wu, Z. Tian, A. Li, and Z. Wang, "Functional immunization of networks based on message passing," Applied Mathematics and Computation, vol. 366, Article ID 124728, 2020.

[31] V. M. Preciado, M. Zargham, C. Enyioha, A. Jadbabaie, and G. Pappas, "Optimal vaccine allocation to control epidemic outbreaks in arbitrary networks," in Proceedings of the 52nd IEEE Conference on Decision and Control, pp. 7486-7491, IEEE, Florence, Italy, December 2013.

[32] L. Böttcher, O. Woolley-Meza, N. A. Araújo, H. J. Herrmann, and D. Helbing, "Disease-induced resource constraints can trigger explosive epidemics," Scientific Reports, vol. 5, Article ID 16571, 2015.

[33] X. Chen, W. Wang, S. Cai, H. E. Stanley, and L. A. Braunstein, "Optimal resource diffusion for suppressing disease spreading in multiplex networks," Journal of Statistical Mechanics: Theory and Experiment, vol. 2018, no. 5, Article ID 053501, 2018.

[34] X. Chen, R. Wang, M. Tang, S. Cai, H. E. Stanley, and L. A. Braunstein, "Suppressing epidemic spreading in multiplex networks with social-support," New Journal of Physics, vol. 20, no. 1, Article ID 013007, 2018.

[35] L. Long, K. Zhong, and W. Wang, "Malicious viruses spreading on complex networks with heterogeneous recovery rate," Physica A: Statistical Mechanics and Its Applications, vol. 509, pp. 746-753, 2018.

[36] J. Jiang, J. Liang, and T. Zhou, "Modeling of epidemic spreading on multilayer networks in uncertain environments," Journal of Statistical Mechanics: Theory and Experiment, vol. 2019, no. 10, Article ID 103404, 2019.

[37] X. Chen, Q. Liu, R. Wang, Q. Li, and W. Wang, "Selfawareness-based resource allocation strategy for containment of epidemic spreading," Complexity, vol. 2020, Article ID 3256415, 12 pages, 2020.

[38] T. Zhou, Q. Liu, Z. Yang et al., "Preliminary prediction of the basic reproduction number of the Wuhan novel coronavirus 2019-nCoV," Journal of Evidence-Based Medicine, vol. 13, no. 1, pp. 3-7, 2020.

[39] L. Wang, J. Wang, H. Zhao et al., "Modelling and assessing the effects of medical resources on transmission of novel coronavirus (covid-19) in Wuhan, China," Mathematical Biosciences and Engineering, vol. 17, no. 4, pp. 2936-2949, 2020.

[40] A. S. Mackie, L. Pilote, R. Ionescu-Ittu, E. Rahme, and A. J. Marelli, "Health care resource utilization in adults with congenital heart disease," The American Journal of Cardiology, vol. 99, no. 6, pp. 839-843, 2007.

[41] M. Gul and A. F. Guneri, "A computer simulation model to reduce patient length of stay and to improve resource 
utilization rate in an emergency department service system," International Journal of Industrial Engineering, vol. 19, no. 5, pp. 221-231, 2012.

[42] M. Girvan and M. E. J. Newman, "Community structure in social and biological networks," Proceedings of the National Academy of Sciences, vol. 99, no. 12, pp. 7821-7826, 2002.

[43] M. Small, Y. Li, T. Stemler, and K. Judd, "Growing optimal scale-free networks via likelihood," Physical Review E, vol. 91, no. 4, Article ID 042801, 2015.

[44] R. Li, L. Dong, J. Zhang et al., "Simple spatial scaling rules behind complex cities," Nature Communications, vol. 8, no. 1, pp. 1-7, 2017.

[45] B. Schönfisch and A. de Roos, "Synchronous and asynchronous updating in cellular automata," BioSystems, vol. 51, no. 3, pp. 123-143, 1999.

[46] P. G. Fennell, S. Melnik, and J. P. Gleeson, "Limitations of discrete-time approaches to continuous-time contagion dynamics," Physical Review E, vol. 94, no. 5, Article ID 052125, 2016.

[47] S.-M. Cai, X.-H. Chen, X.-J. Ye, and M. Tang, "Precisely identifying the epidemic thresholds in real networks via asynchronous updating," Applied Mathematics and Computation, vol. 361, pp. 377-388, 2019.

[48] M. Catanzaro, M. Boguñá, and R. Pastor-Satorras, "Generation of uncorrelated random scale-free networks," Physical Review E, vol. 71, no. 2, Article ID 027103, 2005.

[49] P. Holme and B. J. Kim, "Growing scale-free networks with tunable clustering," Physical Review E, vol. 65, no. 2, Article ID 026107, 2002.

[50] M. Boguná, R. Pastor-Satorras, and A. Vespignani, "Cut-offs and finite size effects in scale-free networks," The European Physical Journal B-Condensed Matter and Complex Systems, vol. 38, no. 2, pp. 205-209, 2004.

[51] P. Shu, W. Wang, M. Tang, and Y. Do, "Numerical identification of epidemic thresholds for susceptible-infected-recovered model on finite-size networks, Chaos," An Interdisciplinary Journal of Nonlinear Science, vol. 25, no. 6, Article ID 063104, 2015.

[52] P. Crepey, F. P. Alvarez, and M. Barthélemy, "Epidemic variability in complex networks," Physical Review E, vol. 73, no. 4, Article ID 046131, 2006.

[53] R. Pastor-Satorras, C. Castellano, P. Van Mieghem, and A. Vespignani, "Epidemic processes in complex networks," Reviews of Modern Physics, vol. 87, no. 3, p. 925, 2015.

[54] M. Barthélemy, A. Barrat, R. Pastor-Satorras, and A. Vespignani, "Velocity and hierarchical spread of epidemic outbreaks in scale-free networks," Physical Review Letters, vol. 92, no. 17, Article ID 178701, 2004.

[55] M. Newman, Networks: An Introduction, Oxford University Press, Oxford, UK, 2010.

[56] S. C. Ferreira, R. S. Sander, and R. Pastor-Satorras, "Collective versus hub activation of epidemic phases on networks," Physical Review E, vol. 93, no. 3, Article ID 032314, 2016.

[57] C. M. O’Connor, A. A. Anoushiravani, M. R. DiCaprio, W. L. Healy, and R. Iorio, "Economic recovery after the covid19 pandemic: resuming elective orthopedic surgery and total joint arthroplasty," The Journal of Arthroplasty, vol. 35, no. 7, pp. S32-S36, 2020.

[58] G. Bonaccorsi, F. Pierri, M. Cinelli et al., "Economic and social consequences of human mobility restrictions under covid19," Proceedings of the National Academy of Sciences, vol. 117, no. 27, pp. 15530-15535, 2020.

[59] R. Pastor-Satorras and A. Vespignani, "Epidemic spreading in scale-free networks," Physical Review Letters, vol. 86, no. 14, p. 3200, 2001. 